\title{
Immunohistochemical Analysis of Sensory Nerve Endings in Ankle Ligaments: A Cadaver Study
}

\author{
Susanne Rein ${ }^{a}$ Elisabet Hagert ${ }^{d} \quad$ Uwe Hanisch $^{c}$ Sophie Lwowski ${ }^{a} \quad$ Armin Fieguth $^{b}$ \\ Hans Zwipp ${ }^{a}$ \\ a Department of Trauma and Reconstructive Surgery, University Hospital 'Carl Gustav Carus', Dresden, \\ b Institute of Pathology, 'Carl Thiem' Hospital, Cottbus, and 'Institute of Legal Medicine, University Hospital of Hannover, \\ Hannover, Germany; ${ }^{d}$ Department of Clinical Science and Education, Hand and Foot Surgery Center, Karolinska \\ Institutet, Stockholm, Sweden
}

\section{Key Words}

Ankle $\cdot$ Immunohistochemistry $\cdot$ Ligaments •

Mechanoreceptors $\cdot$ Proprioception

\begin{abstract}
Background: The aim of this study was to analyze the pattern and types of sensory nerve endings in ankle ligaments using immunohistochemical techniques, in order to gain more insight into functional ankle stability. Methods: One hundred forty ligaments from 10 cadaver feet were included: the calcaneofibular and anterior/posterior talofibular ligaments from the lateral complex; inferior extensor retinaculum complex, talocalcaneal oblique and canalis tarsi ligaments from the sinus tarsi; deltoid ligament with its individual portions from the medial complex, and anterior tibiofibular ligament (ATiFL) from the syndesmosis. Mechanoreceptors were classified according to Freeman and Wyke [Acta Anat (Basel) 1967;68:321-333] after staining with hematoxylin-eosin, low-affinity neurotrophin receptor p75, protein gene product 9.5, and S-100 protein. Results: Free nerve endings were the predominant sensory endings in all four complexes, with the greatest density in the lateral and medial complexes; followed by Ruffini endings, unclassifiable
\end{abstract}

corpuscles, Pacini corpuscles, and Golgi-like endings. Ruffini endings were significantly more prevalent in the ATiFL than in the medial complex, and more common than Pacini corpuscles and Golgi-like endings in the lateral, medial, and sinus tarsi complexes. A greater number of blood vessels correlated with a greater number of free nerve endings. There was a negative correlation between the number of Ruffini endings, unclassifiable corpuscles, and age. Conclusions: Free nerve endings are the dominant mechanoreceptor type in the ankle ligaments, followed by Ruffini endings. The ligaments of the lateral and medial ankle complexes are more innervated than the sinus tarsi ligaments.

Copyright $\odot 2012$ S. Karger AG, Basel

\section{Introduction}

Joint stability relies upon fine interactions of both static and dynamic elements. While static joint stability is constituted by the anatomical congruity of joint surfaces and the ligamentous restraints acting to limit joint translations, the dynamic aspects of joint stability chiefly concern proprioceptive control of the compressive and directional muscular forces acting on a joint [Frank, 2004].

\section{KARGER}

Fax +4161306 1234

E-Mail karger@karger.ch

www.karger.com (c) 2012 S. Karger AG, Basel

1422-6405/13/1971-0064\$38.00/0

Accessible online at:

www.karger.com/cto
PD Dr. Susanne Rein

Department of Trauma and Reconstructive Surgery

University Hospital 'Carl Gustav Carus'

Fetscherstrasse 74, DE-01307 Dresden (Germany)

Tel. +49 351458 2402, E-Mail susanne.rein@web.de 


\section{Abbreviations used in this paper}

\section{Ligaments}

Lateral complex

ATFL anterior talofibular ligament

CFL calcaneofibular ligament

PTFL posterior talofibular ligament

Medial complex

(a) Superficial layer

TNL tibionavicular ligament

TCL tibiocalcaneal ligament

STTL superficial tibiotalar ligament

(b) Deep layer

ATTL anterior tibiotalar ligament

PTTL posterior tibiotalar ligament

Sinus tarsi

IER inferior extensor retinaculum

IERI inferior extensor retinaculum, intermediate root

IERL inferior extensor retinaculum, lateral root

IERM inferior extensor retinaculum, medial root

TCOL talocalcaneal oblique ligament; synonym: cervical ligament

CTL canalis tarsi ligament; synonym: interosseous talocalcaneal ligament

Distal tibiofibular syndesmosis

ATiFL anterior tibiofibular ligament

Further abbreviations

$\mathrm{H} \& \mathrm{E} \quad$ hematoxylin-eosin

HRP horseradish peroxidase

IR immunoreactivity

p75 low-affinity nerve growth factor receptor p75

PBS phosphate-buffered saline

PGP 9.5 protein gene product 9.5

S100 S-100 protein

Ligaments are important structures with a role not only in the static stability of joints but also in the sensorimotor control of joint movements. As such, ligaments are recognized as sensory organs, capable of monitoring and supplying relevant kinesthetic and proprioceptive data [Solomonow, 2006].

Proprioception is a critical part of ankle stability. The stability of the ankle joint is provided by a lateral and medial complex of ligaments as well as the distal tibiofibular syndesmosis [Zwipp, 1994]. The subtalar joint plays a key role in adapting the foot to the ground [Stagni et al., 2003], which is stabilized by the sinus tarsi ligaments [Schmidt, 1978; Zwipp, 1994].

Several studies have investigated sensory nerve endings of ankle ligaments using the gold chloride technique [Michelson and Hutchins, 1995; Moraes et al., 2008]. However, gold chloride impregnates not only nerve tissue but also elastic fibers in blood vessels and reticular fibers, thus providing nonspecific imaging of neural elements in tissue [Soule, 1962; Koch et al., 1995; Gómez-Barrena et al., 1999].

Recently, immunohistochemical analysis precisely determined sensory nerve endings in wrist ligaments [Hagert et al., 2004, 2005, 2007]. This technique utilized specific neural and perineural markers: glial cell S-100 protein (S100), low-affinity p75 neurotrophic receptor (p75), and protein gene product 9.5 (PGP 9.5). The innervation of ligaments is characterized by specific nerve endings, so-called mechanoreceptors, which can be classified due to their typical shape and neurophysiological traits according to Freeman and Wyke [Freeman and Wyke, 1967a]. As different mechanoreceptors have been attributed different functions, a profound analysis of the distribution of sensory nerve endings in ankle-stabilizing ligaments will give more insight into functional ankle stability.

The aim of this study was to analyze the pattern and types of mechanoreceptors in the different anatomical complexes of ankle ligaments using designated immunohistochemical markers, as well as the overall vascularity of the ligaments.

\section{Materials and Methods}

Cadaver Specimens

All protocols in this study were approved by the local ethics committee review board. Ten feet from 5 subjects ( 2 women and 3 men) with a mean age of $57 \pm 20$ years (range $36-86$ years) were included in this study. Five left and 5 right feet were analyzed. The cadavers were refrigerated $\left(4^{\circ} \mathrm{C}\right)$ pending ligament harvest, and the mean time between death and harvest was $3.6 \pm 2.4$ days (range 1-7 days). All feet were assessed macroscopically and showed no signs of ligament injury or structural abnormality.

\section{Ligament Specimens}

A lateral and medial semicircular skin incision was made over the ankle. The lateral and medial ligaments as well as the ligaments of the sinus tarsi were exposed.

Sinus tarsi ligaments were defined according to Schmidt [1978]. Dissection of the different portions of the deltoid ligament was based on the description of Pankovich and Shivaram [1979]. The other ligaments were defined according to the description of Zwipp [1994].

The anterior (ATFL) and posterior talofibular ligaments (PTFL) as well as the calcaneofibular ligament (CFL) were obtained from the lateral complex. The lateral (IERL), intermediate (IERI), and medial (IERM) roots of the inferior extensor retinaculum (IER), the talocalcaneal oblique ligament (TCOL) and the canalis tarsi ligament (CTL) were resected in the sinus tarsi. The tibionavicular (TNL), tibiocalcaneal (TCL), and superficial tibiotalar (STTL) ligaments from the superficial layer, as well as the anterior (ATTL) and posterior tibiotalar (PTTL) portions from the 
deep layer of the deltoid, were harvested from the medial complex. The anterior interosseous tibiofibular ligament (ATiFL) from the distal tibiofibular syndesmosis was also resected. All 140 ligaments were completely dissected from their insertion into bone.

\section{Immunohistochemistry}

Specimens were immediately fixed in $4 \%$ buffered formalaldehyde solution $(\mathrm{pH}=7.4)$ for $24 \mathrm{~h}$ at $4^{\circ} \mathrm{C}$, decalcified with diaminoethanetetraacetic acid (EDTA), and embedded in paraffin. Sections of $4 \mu \mathrm{m}$ were cut and mounted onto silane-coated slides for conventional staining and immunohistochemistry. All ligaments were cut at 5 levels, with a $50-\mu \mathrm{m}$ cutting interval between each level.

The mounted sections were dehydrated beginning with xylol in decreasing concentrations. Sections were then rehydrated with distilled water. Slides were incubated in a $1 \% \mathrm{H}_{2} \mathrm{O}_{2}$ blocked endogenous peroxidase activity for $5 \mathrm{~min}$ at room temperature, and rinsed for $3 \times 5 \mathrm{~min}$ in phosphate-buffered saline (PBS; $\mathrm{pH}=7.4$ ). Subsequently, the slides were treated with an ultra vision horseradish peroxidase-polymer kit (HRP-polymer kit, code TL-060$\mathrm{HL}$; Thermo Scientific, Schwerte, Germany) for $5 \mathrm{~min}$ at $37^{\circ} \mathrm{C}$, followed by incubation with primary antibodies for $60 \mathrm{~min}$ at $37^{\circ} \mathrm{C}$. After rinsing with PBS $3 \times 5 \mathrm{~min}$, the secondary antibody with enhancer of the ultra vision HRP-polymer kit was applied for $10 \mathrm{~min}$ at room temperature. The sections were washed in PBS $3 \times 5$ min again before the HRP-polymer kit was used for $15 \mathrm{~min}$ at room temperature. Afterwards the sections were rinsed in PBS $3 \times 5$ min once again and detected with chromogene 3 -amino9-ethylcarbazol (AEC; Romulin, code REEC810 L; Zytomed Systems; Berlin, Germany) for $15 \mathrm{~min}$ at room temperature.

Thereafter followed rinsing in distilled water, and counterstaining with hematoxylin was performed. Finally, sections were dehydrated and covered with Entellan (Merck, Darmstadt, Germany). As a negative control, identical staining without addition of primary antibodies was performed in parallel followed by counterstaining with hematoxylin. When staining for PGP 9.5 was performed, the slides were initially pretreated with $1 \%$ Triton X-100 solution for $20 \mathrm{~min}$ at room temperature and then rinsed in PBS 3 $\times 5$ min before treatment with $1 \% \mathrm{H}_{2} \mathrm{O}_{2}$, as described above.

\section{Antibodies}

Polyclonal rabbit antisera against p75 (working dilution: 1:200; code N-3908; Sigma, Saint Louis, Mo., USA) was used. The antibody specifically reacts with the low-affinity neurotrophic receptor, which binds nerve growth factor and all other neurotrophins, including brain-derived neurotrophic factor, neurotrophin-3, and neurotrophin-4.

Polyclonal rabbit antisera against PGP 9.5 (working dilution 1:500; code: 7863-0504; AbD Serotec, Düsseldorf, Germany) and polyclonal antisera against S100 (working dilution 1:500; code Z 0311; DakoCytomation, Glostrup, Denmark) were also used. The antibody against PGP 9.5 is a panneuronal marker reacting with PGP 9.5 in all mammalian species tested, including humans. The antibody against S100 specifically stains the S100 protein, including Schwann cells of the peripheral nervous system.

The monoclonal mouse anti-human smooth muscle-actin 1A4 antibody (sm-actin; working dilution 1:750; code M 0851; DakoCytomation) labels smooth muscle cells in blood vessels, whereas epithelia, lymphocytes, cardiac and skeletal muscle cells, endothelial cells, fat cells, Schwann cells, and fibroblasts are negative.

\section{Morphological Analysis and Cell Counting}

Histological examination of the stained tissue sections was performed using a Leica light microscope (Leitz DMRBE, Wetzlar, Germany) with a Leica camera (Leica DC 300; Leica Microsystems CMS GmbH, Heerbrugg, Switzerland).

Hematoxylin-eosin (H\&E)-stained slices were used for determination of tissue morphology, and all ligaments were evaluated to exclude signs of ligament lesions before starting the mechanoreceptor analysis, because ligamentous lesions lead to a gradually decreased number of sensory nerve endings over time [Denti et al., 1994]. In order to exclude acute lesions, histological analysis was focused on determining possible necrosis, bleeding, fibrin exudation, and granulation tissue. For exclusion of old lesions, bleeding residuals, focal intraligamentous vascularization inside the connective collagen tissue, fibroblast proliferation, hyalinization of collagen fibroblasts, scar tissue, chondroid metaplasia outside the insertion, calcification, and ossification were assessed.

Mechanoreceptors were analyzed according to the classification of Freeman and Wyke, modified by Hagert [Freeman and Wyke, 1967b; Hagert, 2008]. Ruffini, Pacini, Golgi-like, and free nerve endings as well as unclassifiable corpuscles were counted in the S100, p75, and PGP 9.5 stainings in all 5 levels with respect to the total cell count per section at an original magnification of $400 \times$ (high-power field). A standard $10 \times 10$ grid was used for determination of mechanoreceptor size. Blood vessels were counted at two representative levels in the sm-actin stain, identified by specific immunoreactivity (IR) of sm-actin of the smooth muscle cells in the wall of the vessels. All specimens were blinded for cell counts.

Sensory corpuscles, which could neither clearly be defined as Ruffini, Pacini, or Golgi-like corpuscles nor as nerve fascicles, were deemed unclassifiable according to Hagert et al. [2005; Hagert, 2008, 2010].

\section{Statistical Analysis}

Means with standard deviations were used for descriptive statistics throughout the article except for figure 4 and table 1, which are reported as absolute values.

The first purpose was to investigate the general distribution of mechanoreceptors and blood vessel in the ligaments. Contingency tables with Fisher's exact test were used for this analysis. The level of significance was considered high with $\mathrm{p} \leq 0.05$.

The second purpose was to analyze the general distribution of sensory nerve endings in all ligaments $(n=140)$, which was performed with the Friedman test followed by the Wilcoxon test with post hoc Bonferroni adjustments. The five different types of mechanoreceptors allowed ten possible test comparisons, which resulted in a significance level of $\mathrm{p} \leq 0.005$.

The third purpose was to analyze the mechanoreceptors between the anatomical complexes. The ATiFL as a part of the distal tibiofibular syndesmosis, and the lateral, medial, and sinus tarsi complexes were compared. The Kolmogorov-Smirnov test was performed to investigate data distribution. Since all groups were found to not have normal distributions, the subsequent statistical analysis was performed using the Kruskal-Wallis test followed by the Mann-Whitney test with post hoc Bonferroni adjustments, with a final level of significance of $p \leq 0.0083$, due to six tests of significance between the four groups at the Bonferroni adjustment. 
Table 1. Exclusion of posttraumatic ligamentous lesions

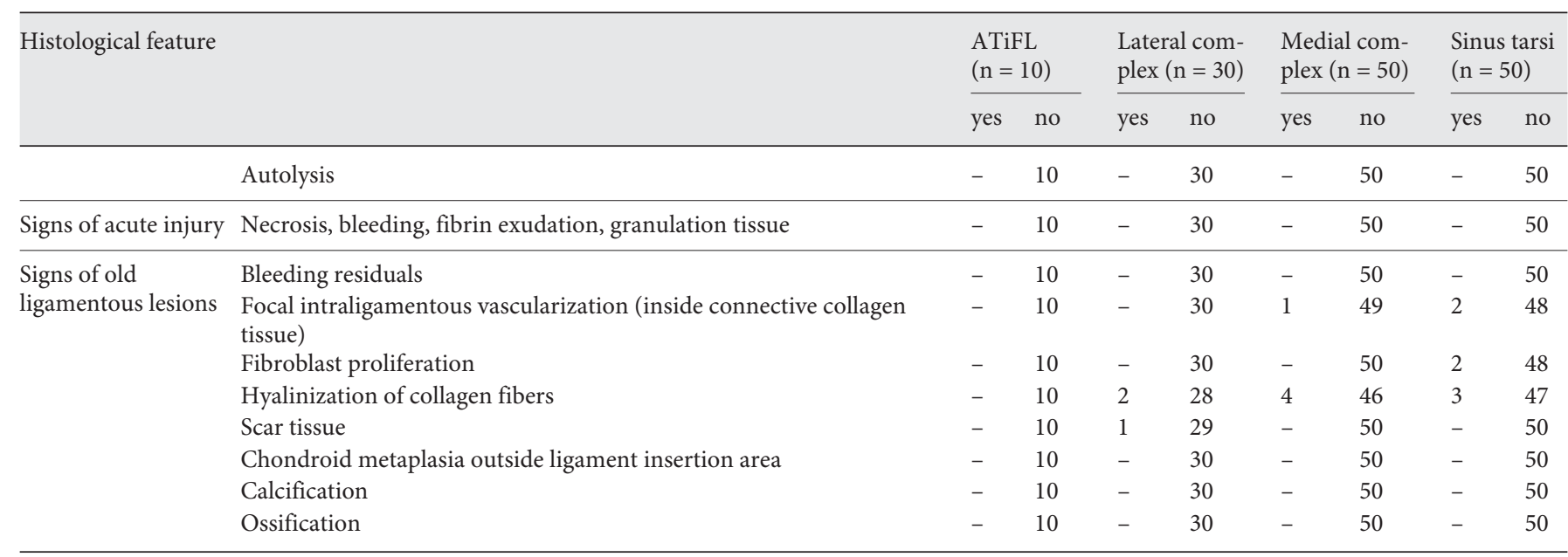

Results of the tissue morphology analysis in H\&E staining are presented. In terms of histological signs of old ligamentous lesions, only few focal and small herd (not larger than one high-resolution visual field) reparative or scar tissue changes were observed, which can be interpreted as an expression of degeneration or state after microtrauma. There, the general/overall ligamentous structure was intact. No morphological correlate for an old complete or partial ligament rupture was seen.

The fourth purpose was to compare the quantity of the different types of mechanoreceptors within each anatomical complex. The Friedman test, followed by the Wilcoxon test with post hoc Bonferroni adjustments, was performed. The final level of significance was $\mathrm{p} \leq 0.005$, since ten tests of significance between the five mechanoreceptor groups were performed at the Bonferroni adjustment.

The fifth purpose was to test correlations with regard to age, sensory nerve endings, and blood vessel numbers. Correlation analysis was performed with Spearman's rho coefficient with a two-sided significance level of $\mathrm{p} \leq 0.05$. All 140 ligaments were examined together for the correlation analysis.

\section{Results}

Acute and chronic lesions were excluded in all ligaments following analysis using H\&E (table 1).

\section{Ruffini Endings (Type I)}

The central axon was clearly demarcated via staining for PGP 9.5 and S100, whereas it showed no specific IR in staining for p75 (fig. 1). The thin and incomplete capsule was IR for p75. Occasionally, identification of smaller corpuscles located within the capsule of corpuscles was also noted. The dendritic nerve terminals within the corpuscle showed IR for S100, p75, and PGP 9.5. Sometimes Ruffini endings were grouped as two endings together. The size of a single Ruffini ending varied between 50 and
$120 \mu \mathrm{m}$. One hundred eight of the 140 ligaments (77\%) contained Ruffini endings.

\section{Pacini Corpuscles (Type II)}

The characteristic lamellar and thick capsule of the $\mathrm{Pa}$ cini corpuscles displayed intense p75 IR. There was a strong intracorpuscular reaction after processing for $\mathrm{S} 100$. Nerve fibers of the afferent nerve fascicle, the so-called parent axon, as well as thin nerve fibers within the corpuscles, were demarcated after staining for PGP 9.5 (fig. 2). Pacini corpuscles were generally smaller than Ruffini endings. They were observed in 49 of 140 ligaments (35\%).

\section{Golgi-Like Endings (Type III)}

Golgi-like endings were rarely found. They were only present in 15 ligaments (11\%), with a total of 28 Golgi-like endings in all 140 investigated ligaments.

They had a large fusiform body with a thin capsule and smaller grouped corpuscles seen within the large body. The smaller corpuscles contained terminal nerve endings with S100, p75, and PGP 9.5 IR. Thick parent axons were seen to enter the corpuscle at one side (fig. 3). The longitudinal axis of the Golgi-like endings was arranged in parallel to the ligamentous collagen bundle composition. Golgi-like endings were generally remarkable due to their size, which considerably varied between 150 and $800 \mu \mathrm{m}$ for the true corpuscle. 
Fig. 1. A Ruffini corpuscle in the IERL in the sinus tarsi as stained with H\&E (a), or for $\mathrm{S} 100$ (b), p75 (c), and PGP 9.5 (d). Note the specific IR of the dendritic terminal nerve endings (b-d) and thin, at times partial, encapsulation (arrowhead) of the corpuscle (c). The central axon (arrow) of the corpuscle is clearly visualized by showing specific S100 IR (b) and PGP 9.5 IR (d). No IR of the central axon is seen in the p75 staining (c). Due to its characteristic dendritic nerve endings, the Ruffini corpuscle is also unspecifically stained and visible in the H\&E staining (a).

Fig. 2. A Pacini corpuscle in the PTFL as seen in H\&E staining (a), with S100 (b), with p75 (c), and with PGP 9.5 IR (d). The central axon (arrow) of the afferent nerve is clearly delineated with $S 100$ (b). Note the characteristic multilamellar capsule (arrowhead) with strong p75 IR in the inner and outer lamellae (c) and PGP 9.5 IR in the inner lamellae (arrowhead in d). Due to its characteristic onion-layered capsule, the Pacini corpuscle is also visible in the unspecific H\&E staining (a).
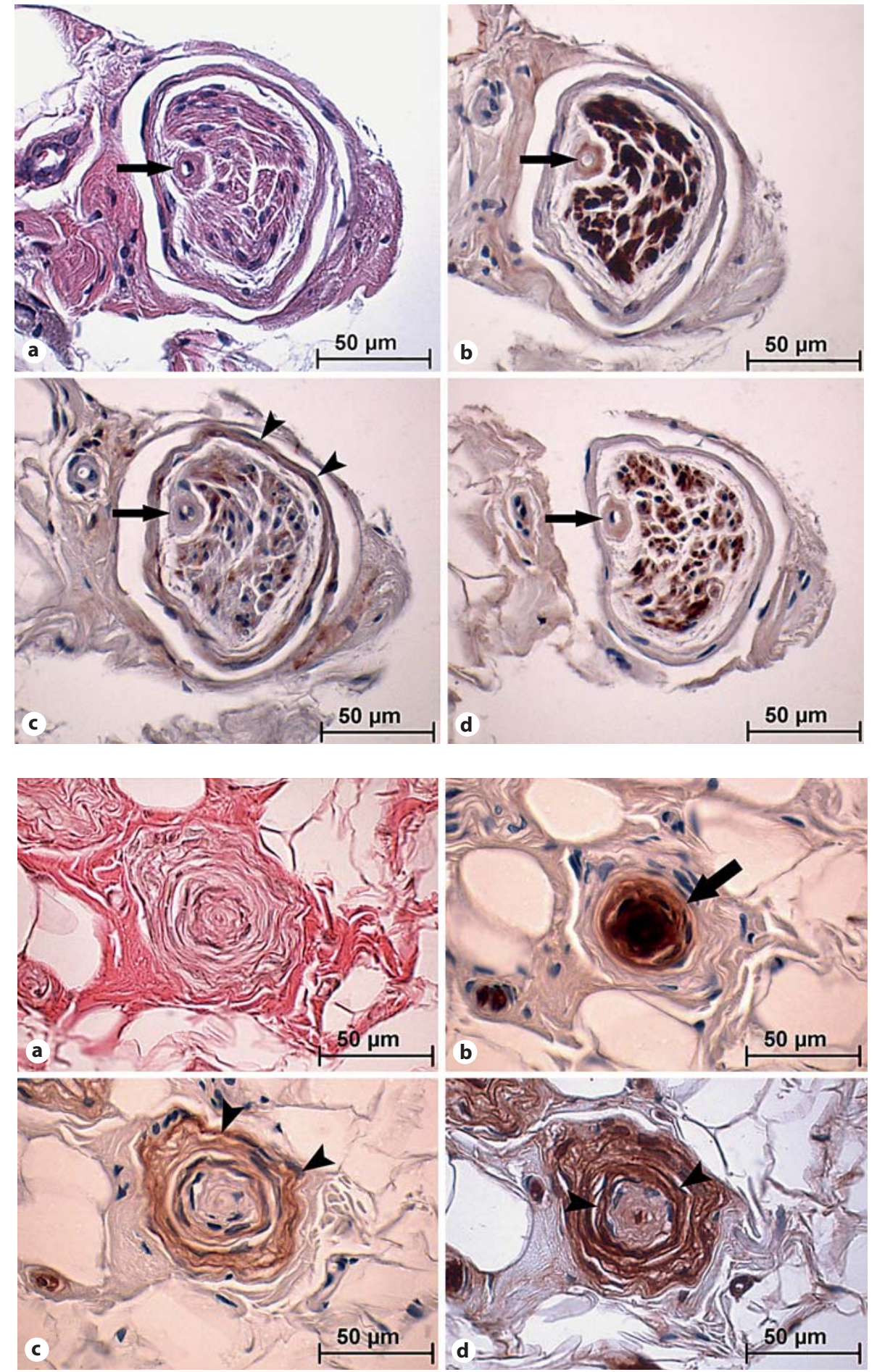

\section{Free Nerve Endings (Type IV)}

Nerve fascicles were generally observed in the vicinity of vessels and in the connective tissue sheaths within the ligaments as well as in the epiligament. There was marked p75 and S100 IR in the nerve fascicle, whereas the axons within the nerve fascicles were stained for PGP 9.5. The size of the free nerve endings varied depending on the degree of myelinization, the number of axons, and the cutting level. All investigated ligaments (100\%) contained free nerve endings. 
Fig. 3. A Golgi-like corpuscle in an IERI is shown as stained with H\&E (a), for S100 (b), p75 (c), and PGP 9.5 (d) IR. An afferent nerve fascicle (arrow) courses to the center of the corpuscle. Smaller corpuscles within the Golgi-like corpuscle are seen, each of them containing terminal nerve endings (arrowheads in $\mathbf{b}-\mathbf{d}$ ).
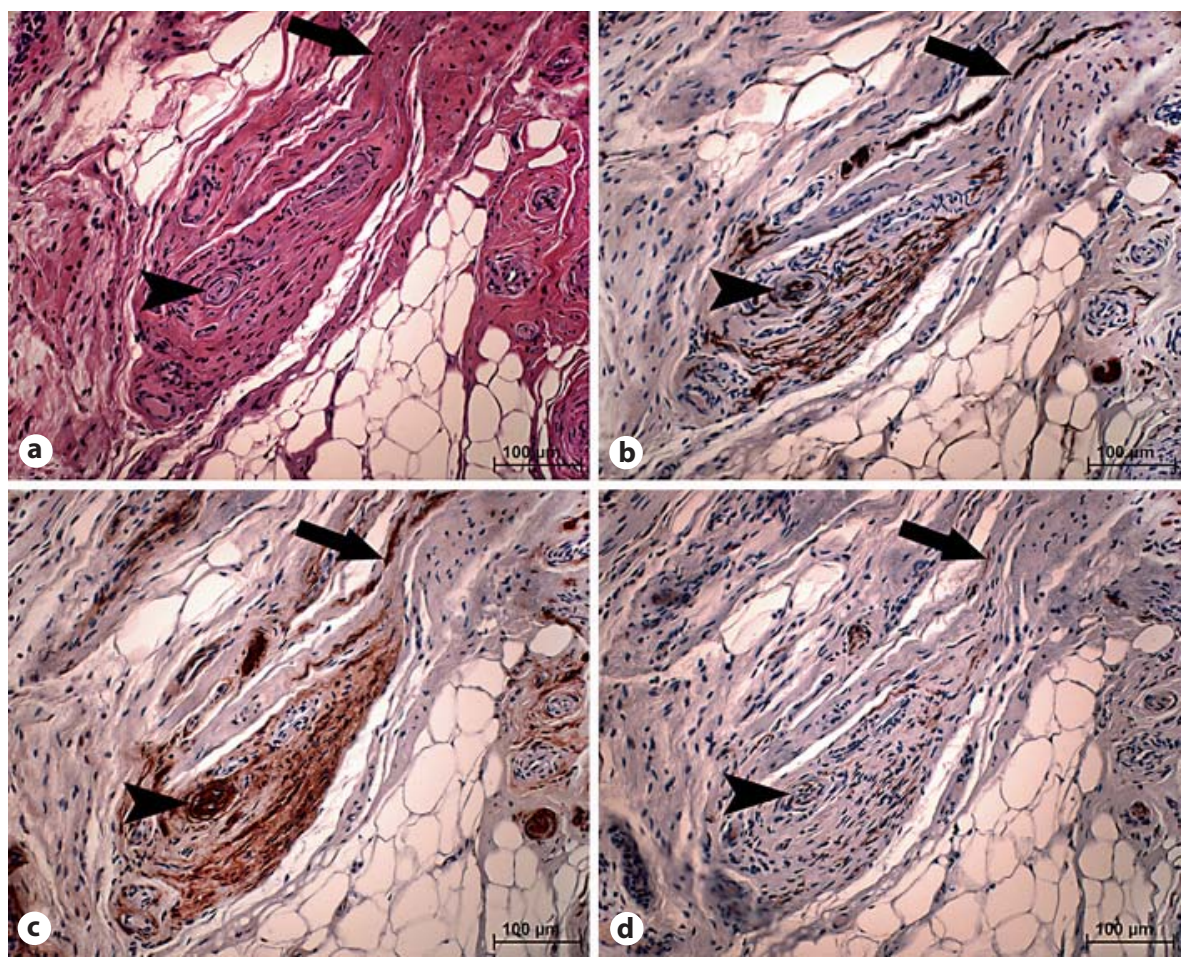

Unclassifiable Corpuscles (Type V)

Certain sensory corpuscles could neither be clearly defined as Ruffini, Pacini, Golgi-like nor as nerve fascicles and were therefore deemed unclassifiable. A variation of size and appearance has been observed. An incomplete capsule was often seen after staining for p75. Unclassifiable corpuscles were documented in 95 of 140 ligaments $(68 \%)$.

\section{General Distribution $(n=140)$}

Sensory nerve endings and blood vessels were equally distributed and significantly more common in the epiligamentous regions compared to the interstitial area ( $p<0.0001$, respectively) (fig. 4). Furthermore, sensory nerve endings were significantly more common close to the insertion into bone rather than the central, or midportion, of a ligament $(\mathrm{p}=0.001)$ or equal distribution. Interestingly, blood vessels were significantly more often interstitial $(\mathrm{p}=0.043)$ and at the insertion into the bone $(\mathrm{p}<0.0001)$ than equally dispersed in the ligament (fig. 4).

The free nerve endings were the predominant sensory ending, followed by Ruffini endings, unclassifiable corpuscles, Pacini corpuscles, and ultimately Golgi-like endings (fig. 5). Statistical analysis showed significant differences between all sensory nerve endings $(p<0.0001$, respectively), except between Ruffini endings and un- classifiable corpuscles. The calculated relationship between the sensory nerve endings is shown in table 2.

No Ruffini, Pacini, or Golgi-like corpuscles were found in 24 of 140 ligaments (17\%). Only free nerve endings were observed in 12 ligaments (8.5\%) (lateral complex: 1 ATFL and 3 CFL; medial complex: 1 TNL, 1 STTL, 1 ATTL, and 1 PTTL; sinus tarsi: 1 IERL, 2 IERM, and 1 $\mathrm{CTL})$. Only free nerve endings and unclassifiable corpuscles were seen in 12 ligaments (8.5\%) (lateral complex: 1 ATFL and 1 PTFL; medial complex: 3 TCL and 2 PTTL; sinus tarsi: 1 IERL, 1 IERM, 2 TCOL, and 1 CTL).

\section{Distribution of Mechanoreceptors between the Anatomical Complexes}

There were no discernible differences in the general morphology of corpuscles between the anatomical complexes. The ATiFL ligament had significantly more Ruffini corpuscles than the medial complex ( $\mathrm{p}=0.005$; fig. 6 ). Significantly more free nerve endings were counted in the lateral complex as compared to the sinus tarsi $(\mathrm{p}<0.0001$; fig. 7). Also, significantly more blood vessels were found in the lateral complex as compared to the sinus tarsi $(\mathrm{p}=$ 0.006 ) and the ATiFL ( $p=0.002$; fig. 7). Furthermore, the medial complex had significantly more free nerve endings $(p<0.0001)$ and blood vessels $(p=0.002)$ than the sinus tarsi (fig. 7). 
Fig. 4. Descriptive distribution of sensory nerve endings and blood vessels in all investigated ligaments $(n=140)$, shown as absolute values. Significantly more sensory endings are found in the epiligamentous region $\left(^{*}\right)$ than interstitially $(\mathrm{p}<0.0001)$ or equally dispersed in the ligament $(\mathrm{p}=$ 0.029). In addition, sensory nerve endings were significantly more often located at the ligament insertion into bone $\left(^{*}\right)$ in comparison with a central $(\mathrm{p}=0.001)$ or equal distribution $(\mathrm{p}<0.0001)$. Blood vessels were similarly significantly more epiligamentous $(+)$ than interstitial or equal $(\mathrm{p}<0.0001$, respectively), and more often found interstitially $(\$)$ than as an equal ligamentous distribution $(\mathrm{p}=0.043)$. Blood vessels (\#) were also significantly more often close to the ligament insertion into bone than equal distributed ( $p<0.0001)$.

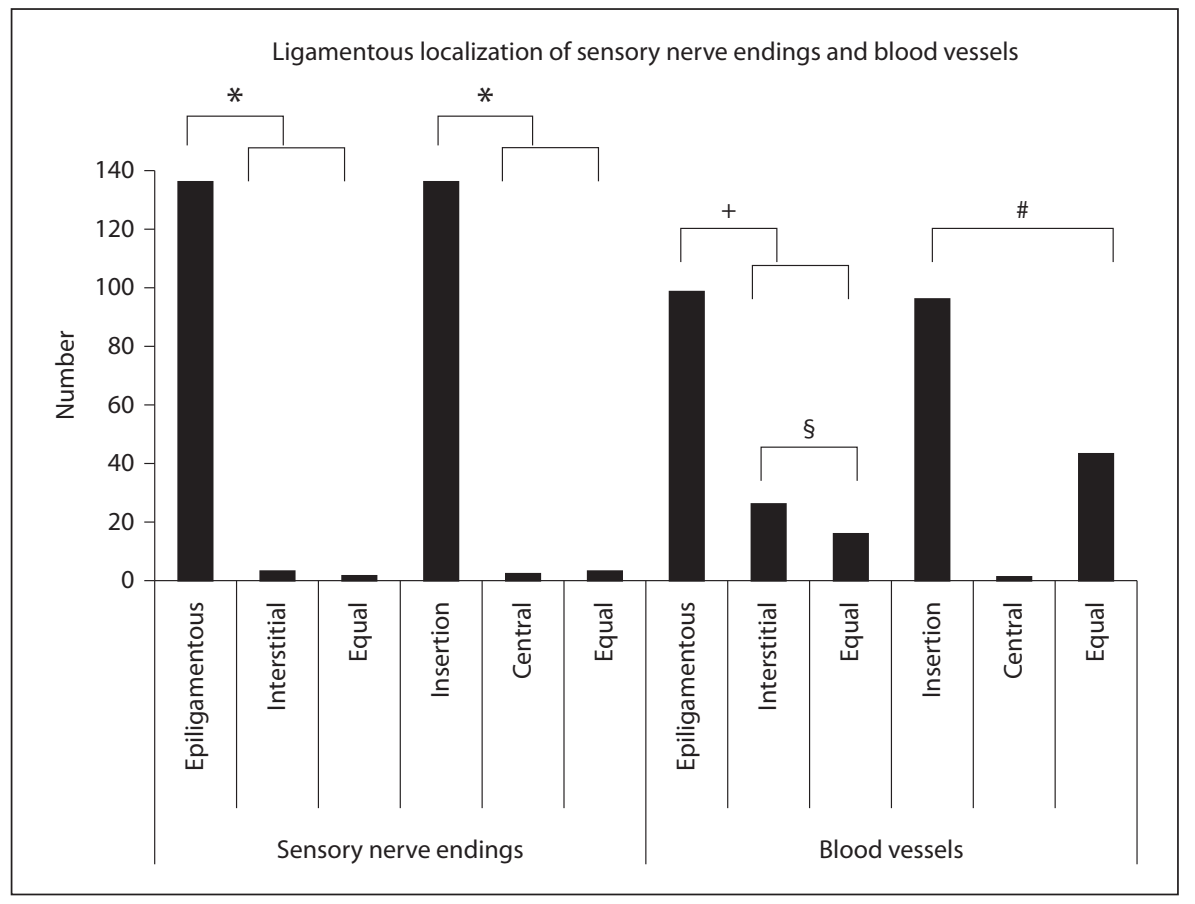

Table 2. Relationship between sensory nerve endings

\begin{tabular}{lc}
\hline Sensory nerve endings & Relationship \\
\hline Ruffini:Pacini & $4.7: 1$ \\
Ruffini:Golgi & $15.5: 1$ \\
Ruffini:unclassifiable & $1.3: 1$ \\
Pacini:Golgi & $3.3: 1$ \\
Pacini:unclassifiable & $1: 3.7$ \\
Golgi:unclassifiable & $1: 12$ \\
Free nerve endings:Ruffini & $122: 1$ \\
Free nerve endings:Pacini & $582: 1$ \\
Free nerve endings:Golgi & $1,890: 1$ \\
Free nerve endings:unclassifiable & $158: 1$ \\
Free nerve endings:blood vessels & $1: 3.6$
\end{tabular}

The relationship between the sensory nerve endings was calculated with the mean of all ligaments $(n=140)$.

\section{Distribution of Mechanoreceptors within the \\ Anatomical Complexes}

Significantly more Ruffini endings than Pacini corpuscles were found in the lateral, medial, and sinus tarsi complexes ( $\mathrm{p}<0.0001$, respectively) (fig. 6). Similarly, significantly more Ruffini endings than Golgi-like endings were found in the lateral, medial, and sinus tarsi complexes ( $\mathrm{p}<0.0001$, respectively) (fig. 6$)$.

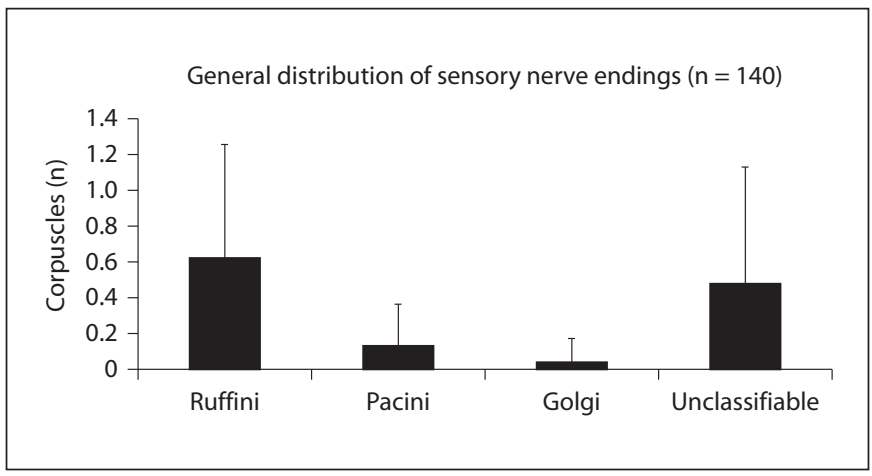

Fig. 5. The general distribution of sensory nerve endings is shown as means with standard deviations. There were significant differences between all sensory corpuscles ( $\mathrm{p}<0.0001$, respectively), except for the comparison between Ruffini-endings and unclassifiable corpuscles.

Significantly more unclassifiable corpuscles than Pacini corpuscles were observed in the lateral $(\mathrm{p}=0.002)$, medial $(\mathrm{p}=0.004)$, and sinus tarsi complexes $(\mathrm{p}<0.0001)$ (fig. 6).

Significantly more unclassifiable corpuscles than Golgi-like endings were observed in the lateral, medial, and sinus tarsi complexes ( $\mathrm{p}<0.0001$, respectively) (fig. 6). 
Fig. 6. Means with standard deviation of mechanoreceptors in the anatomical complexes are shown. The ATiFL (*) had significantly more Ruffini endings than the medial complex $(p=0.005)$. Significantly more Ruffini endings $(\$)$ were found as compared to Pacini corpuscles in the lateral, medial, and sinus tarsi complexes ( $p<0.0001$, respectively). Significantly fewer Golgi-like endings (\#) than Ruffini endings and unclassifiable corpuscles were observed in the lateral, medial, and sinus tarsi complexes $(\mathrm{p}<0.0001$, respectively). Significantly fewer Pacini (lateral: $\mathrm{p}=0.002$; medial: $\mathrm{p}=0.004$; sinus tarsi: $\mathrm{p}<0.0001)$ and Golgi-like endings $(\mathrm{p}<$ 0.0001 , respectively) than unclassifiable corpuscles $(+)$ were seen in the lateral, medial, and sinus tarsi complexes.

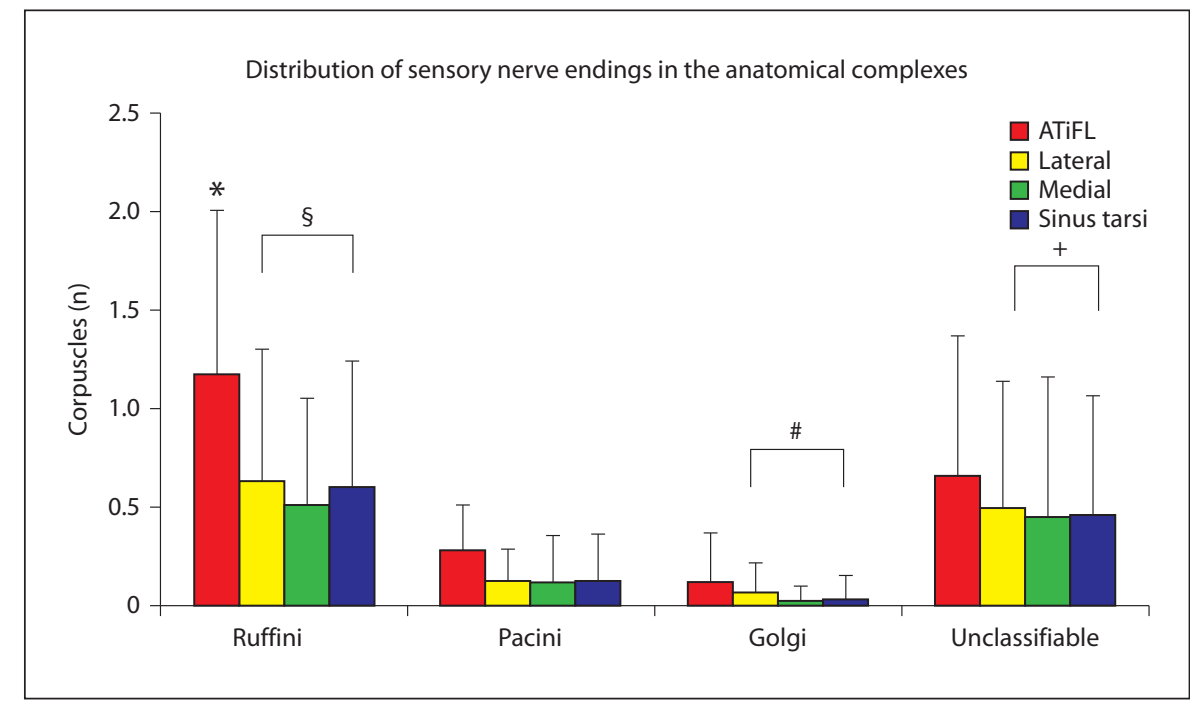

Finally, significantly more free nerve endings than Ruffini, Pacini, Golgi-like, and unclassifiable corpuscles were seen in the lateral, medial, and sinus tarsi complexes $(\mathrm{p}<0.0001$, respectively) as well as the ATiFL ( $\mathrm{p} \leq$ 0.005 , respectively).

\section{Correlation Analysis}

A greater number of blood vessels correlated significantly with a greater number of free nerve endings $(\mathrm{p}<$ $0.0001 ; \mathrm{r}=0.57$; fig. 8). There was also a significant positive correlation between the number of Ruffini endings and the number of free nerve endings $(\mathrm{p}=0.006 ; \mathrm{r}=0.23)$ as well as unclassifiable corpuscles $(\mathrm{p}<0.0001 ; \mathrm{r}=0.43$ ). In addition, there was a significant positive correlation between the number of Pacini corpuscles and Golgi-like endings ( $\mathrm{p}=0.05 ; \mathrm{r}=0.17$ ).

There was a significant negative correlation between Ruffini endings ( $\mathrm{r}=-0.33$; fig. 9), as well as unclassifiable corpuscles $(\mathrm{r}=-0.44$; fig. 10$)$ and age, with a decrease in innervation in older specimens ( $\mathrm{p}<0.0001$, respectively).

\section{Discussion}

\section{General Distribution}

In our study, sensory corpuscles and blood vessels were found mainly close to ligament insertions into bone as well as in the epiligamentous region of ligaments. This is in accordance with previous publications, which also describe the majority of mechanoreceptors at the insertion of ligaments in the shoulder [Morisawa, 1998], wrist

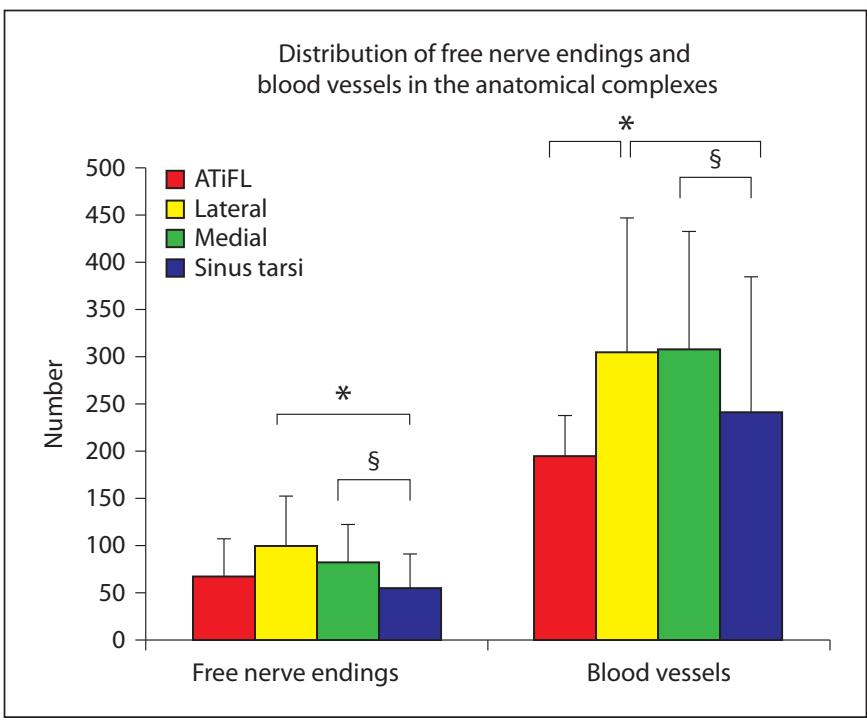

Fig. 7. Means with standard deviations for the free nerve endings and blood vessels are shown. The lateral complex $\left(^{*}\right)$ had significantly more free nerve endings $(\mathrm{p}<0.0001)$ than the sinus tarsi as well as more blood vessels as compared to the sinus tarsi ( $\mathrm{p}=$ $0.006)$ and ATiFL $(p<0.002)$. Furthermore, the medial complex $(\$)$ had significantly more free nerve endings $(\mathrm{p}<0.0001)$ and blood vessels $(p<0.002)$ than the sinus tarsi.

[Hagert et al., 2007; Tomita et al. 2007], knee [Kennedy et al., 1982; Schultz et al., 1984; Schutte et al., 1987; Del Valle et al., 1998], and ankle joints [Moraes et al., 2008]. Similarly, $93 \%$ of the mechanoreceptors in cat lateral ankle ligaments have been found near the attachment to the fibula and calcaneus [Takebayashi et al., 1997]. Krauspe 
Fig. 8. A higher number of blood vessels significantly correlated with a higher number of free nerve endings $(\mathrm{p}<0.0001$; $\mathrm{r}=0.57)$.

Fig. 9. The number of Ruffini endings significantly decreased with older age $(\mathrm{p}<$ $0.0001 ; \mathrm{r}=-0.33$ ).

Fig. 10. The number of unclassifiable corpuscles significantly decreased with older age $(\mathrm{p}<0.0001 ; \mathrm{r}=-0.44)$.
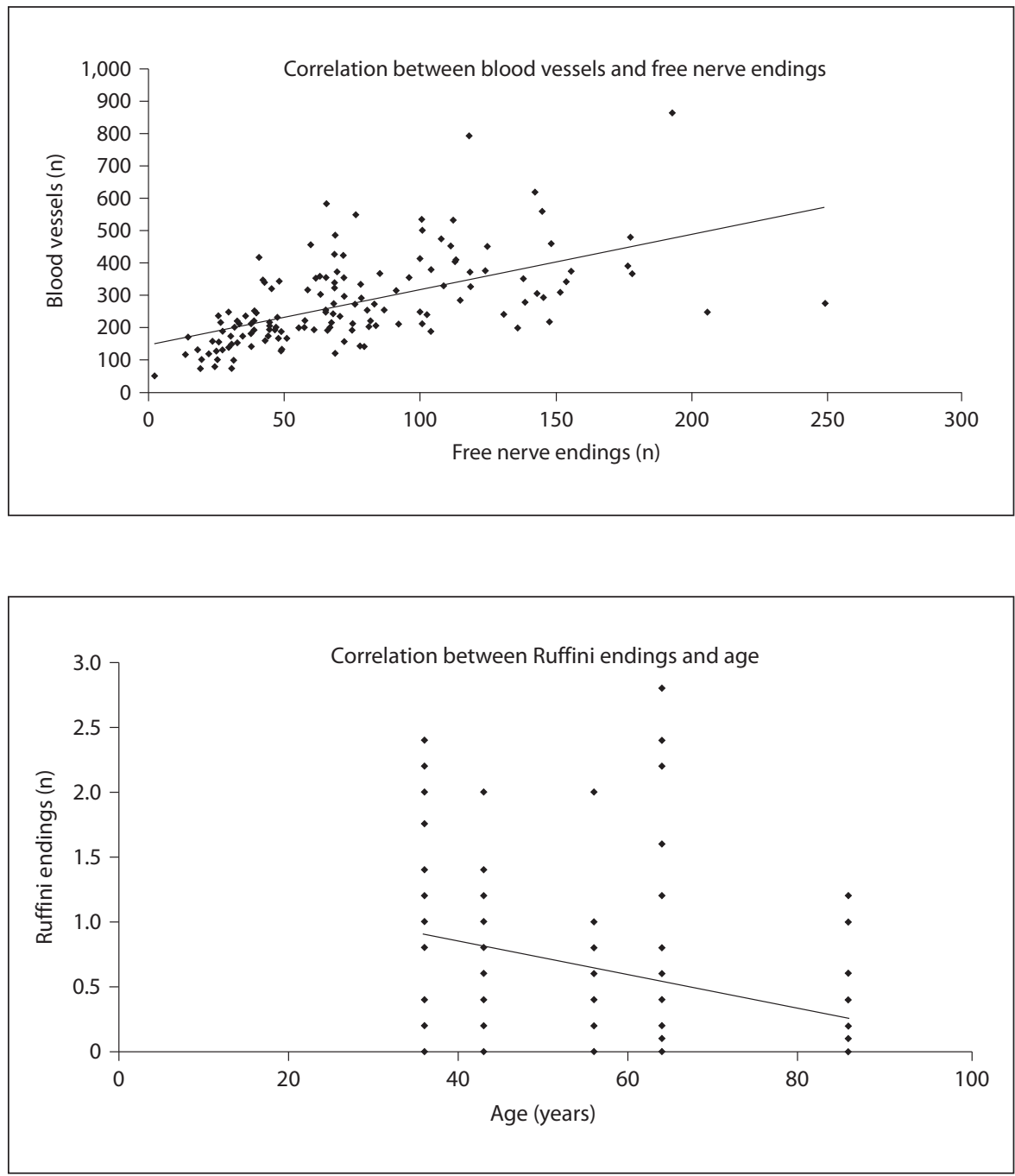

Correlation between unclassifiable corpuscles and age

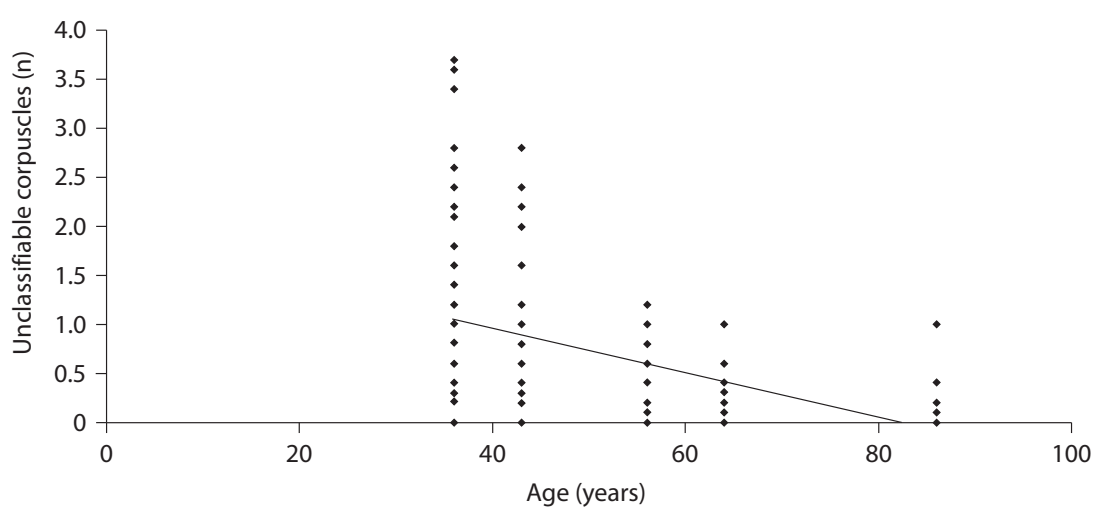


et al. [1992] also reported that a majority of mechanoreceptors in the cat anterior cruciate ligament were found near its femoral attachment. In human knees mechanoreceptors are located near the surface of the ligament, equivalent to the epiligamentous region described above [Schultz et al., 1984]. This epiligamentous area has been proposed to be of importance for ligament nutrition and protection, as well as for providing support to the neurovascular bundles in the ligament [Chowdhury et al., 1991]. A polar distribution of mechanoreceptors allows them to act more sensitively as monitors of tension applied to the ligament as compared to those situated in the center of a ligament [Takebayashi et al., 1997]. Since ligaments are more resistant to strains close to their insertions, this serves to ensure triggering of sensory nerve endings only by potentially noxious motions, while they remain silent during ordinary joint activity [Solomonow, 2006].

Disruption of lateral ankle ligaments mostly occurs intraligamentously [Zwipp, 1986], indicating a preservation of vascularity and sensory nerve endings at the bony insertions which is known to have a positive effect on ligament healing [Ivie et al., 2002]. Immobilization of the ankle, both for conservative and for postoperative treatment, generally leads to healing of a disrupted ligament. If the ligament tensile strength is restored, sensory reeducation with proprioceptive exercises can be initiated to stimulate the remaining sensory nerve endings at the bony insertion and enhance proprioceptive function of the ankle ligaments [Freeman et al., 1965; Cooper and Farr, 1978; Bernier and Perrin, 1998]. The ligamentous insertion regions should thus be conserved during surgery to avoid iatrogenic lesions of important sensory nerve endings [De Avila et al., 1989].

Free nerve endings were the predominant mechanoreceptor type seen in the ankle ligaments, followed by Ruffini endings, unclassifiable corpuscles, Pacini corpuscles, and Golgi-like endings. In relation to the proprioception of the ankle joint it indicates an importance of reacting to noxious, chemical, mechanical, and inflammatory stimuli. The subsequent predominance of Ruffini endings serves to maintain control of joint position and kinesthesia. Joint acceleration/deceleration, as detected by Pacini corpuscles, appears to play only a minor role in ankle joint proprioception. Analogous results were found in recent immunohistological analysis of wrist ligaments, where Ruffini endings were seen more often than Pacini corpuscles [Hagert et al., 2004, 2005, 2007]. Only few Golgi-like corpuscles have been found in the whole analysis. This is in contrast with previous literature, where a predominance of Pacini corpuscles, followed by Golgilike corpuscles, and lastly Ruffini corpuscles, were described in the deltoid ligament and/or lateral ankle ligaments using the gold chloride technique [Michelson and Hutchins, 1995; Moraes et al., 2008]. In addition, as Golgi-like endings detect extreme ranges of joint movement [Newton, 1982], they appear more often in ligaments of big joints, e.g. the cruciate ligament of the knee [Johansson et al., 1991], than in ligaments of small joints [Lin et al., 2006; Hagert, 2008; Chikenji et al., 2010].

The innate function and physiological properties of unclassifiable corpuscles is presently unknown. A pure morphological analysis of unclassifiable corpuscles can never deduce a physiological function - rather, a microneurographic investigation would be of importance to further our knowledge of this enigmatic sensory nerve ending. The high number of unclassifiable corpuscles seen in our study is an expression of the limitations of an applied descriptive analysis using 2D light-microscopy, which does not permit a complete representation of the whole mechanoreceptor. The primary difference between our study and those published previously is that we have used a combination of immunohistochemical markers to target specific mechanoreceptor structures, and we performed the total analysis on a large number of sections at five different levels in each ligament. We propose that our analysis offers a greater distinction of both receptor type and frequency based on this. However, immunofluorescence analysis offers a 3D analysis of sensory nerve endings and is frequently used by researchers [Hagert et al., 2012; Lee et al., 2012]. Nevertheless, even 3D reconstructions of multiple levels of ligaments have revealed a great portion of unclassifiable corpuscles in wrist ligaments [Tomita et al., 2007], which points to a possible limitation of the existing gold-standard classification of Freeman and Wyke, modified by Hagert [Freeman and Wyke, 1967a, b; Hagert, 2008].

Few ligaments have only had free nerve endings without Ruffini, Pacini, or Golgi-like corpuscles. However, no general pattern of particular ligaments of this could be observed, because ligaments from all anatomical complexes were involved.

\section{Anatomical Complexes}

In our study, Ruffini endings were the second most common receptor overall, and they were particularly frequent in the ATiFL. Studies on the biomechanical function of the ATiFL has shown that once the ATiFL is cut, the tibia and lateral malleolus can move separately [Teramoto et al., 2008]. The distal tibiofibular syndesmosis at- 
taches the fibula dynamically at the tibia, which gives the fibula the possibility to perform a three-dimensional movement during physiological dorsalextension and plantarflexion of the ankle joint [Close, 1956; Reimann and Anderhuber, 1980; Peter et al., 1994; Beumer et al., 2003; Rammelt et al., 2008]. The function of the Ruffini ending in the ATiFL is thus to provide constant monitoring of the joint position and motion of this important articulation, and corresponds well with our frequent findings of Ruffini endings in this region.

The lateral and medial complexes are richly innervated by free nerve endings, whereas the sinus tarsi is less so. It seems, therefore, that the ligaments of the lateral and medial complexes as the primary stabilizers of the ankle joint have more capacity for the detection of proprioceptive information than the sinus tarsi ligaments.

In 1965, Freeman et al. [1965] were the first to introduce the term 'functional instability' as a patient's subjective feeling that the ankle is giving way, meaning that a patient has the feeling of instability of the ankle, particularly when walking or running on uneven ground [Freeman et al., 1965]. It has been hypothesized that this proprioceptive deficit may result from a partial deafferentation caused by damage of sensory nerve endings of the joint capsule and ligaments, resulting in functional instability of the ankle [Freeman et al., 1965]. The latter could lead to loss of position sense [Glencross and Thornton, 1981], delayed peroneal reaction time [Konradsen and Ravn, 1991; Karlsson and Andreasson, 1992], strength deficits, and impaired postural control [Tropp and Odenrick, 1988; Richie, 2001; Hertel, 2002; Arnold et al., 2009; Munn et al., 2010]. This innervation study shows that nociception, mediated by free nerve endings, has enormous importance in ankle proprioception, followed by joint position sense due to a predominant occurrence of Ruffini endings.

\section{Correlation Analysis}

We found a positive correlation between the degree of vascularity and innervation, corresponding with previous studies on human ligaments [Chowdhury et al., 1991; Hagert et al., 2004, 2005, 2007]. Similarly, there was a positive correlation between Ruffini endings and free nerve endings or unclassifiable corpuscles, while the presence of Pacini corpuscles was positively correlated with that of Golgi-like endings. As discussed above, mechanoreceptors are mainly located in the epiligament and the ligamentous insertion into bone. The insertion region of a ligament is particularly exposed to a complex combination of both compressive and tensile forces
[Spalazzi et al., 2006]. The presence of a multitude of receptor types in this region thus permits sensorimotor monitoring of all joint activities, where different mechanoreceptor types complement each other due to their different physiological properties, guaranteeing sensorimotor control of all complex joint movements. This ligament-bone interface thus has ideal anatomical and physiological properties for joint protective sensorimotor functions.

The presence of Ruffini endings had a negative correlation with age, with a decrease in Ruffini endings in older specimens. Although these results have a limitation in that they are based on a small sample size, the finding is interesting as it is in agreement with previous studies on animals where a general decrease in neurotrophins has been correlated with the reduction of sensory nerves that occurs in senescence [Ulfhake et al., 2002], and the total number of Ruffini endings in the rabbit anterior cruciate ligament has been found to significantly decrease with aging [Aydog et al., 2006]. These results also correlate with clinical observations on functional ankle stability, where older age has been associated with increased peroneal reaction time and impaired balance control [Rein et al., 2010]. A decrease in sensory nerve endings with older age may thus contribute to the lack of balance and coordination observed in elderly individuals, resulting in an increased risk of fall and injury [Runge, 2002; Kwan et al., 2011].

In conclusion, sensory nerve endings are mainly located closed to the ligament insertion into bone and in the epiligamentous region. Free nerve endings were the predominant receptor type, followed by Ruffini endings, indicating that nociception and joint position have enormous importance in ankle proprioception. The ligaments of the lateral and medial ankle complexes are more innervated than the sinus tarsi ligaments, which implies more capacity for detection of proprioceptive information in these ligaments. A decrease in sensory nerve endings with age has been observed.

\section{Acknowledgements}

This study was financially supported by the Medical Faculty of the Technical University, Dresden, Germany.

The authors thank the following individuals for their contributions to this article: Ursula Range for statistical support, Thomas Albrecht for photographical work, Suzanne Manthey for histological preparation, and Reinhold Krentscher and Hans-Dieter Tröger for logistical support. 


\section{References}

Arnold, B.L., S.W. Linens, S.J. de la Motte, S.E. Ross (2009) Concentric evertor strength differences and functional ankle instability: a meta-analysis. J Athl Train 44: 653-662.

Aydog, S.T., P. Korkusuz, M.N. Doral, O. Tetik, H.A. Demirel (2006) Decrease in the numbers of mechanoreceptors in rabbit ACL: the effects of ageing. Knee Surg Sports Traumatol Arthrosc 14: 325-329.

Bernier, J.N., D.H. Perrin (1998) Effect of coordination training on proprioception of the functionally unstable ankle. J Orthop Sports Phys Ther 27: 264-275.

-Beumer, A., E.R. Valstar, E.H. Garling, R. Niesing, J. Ranstam, R. Löfvenberg, B.A. Swierstra (2003) Kinematics of the distal tibiofibular syndesmosis: radiostereometry in 11 normal ankles. Acta Orthop Scand 74: 337-343.

Chikenji, T., D. Suzuki, M. Fujimiya, T. Moriya, S. Tsubota (2010) Distribution of nerve endings in the human proximal interphalangeal joint and surrounding structures. J Hand Surg Am 35: 1286-1293.

Chowdhury, P., J.R. Matyas, C.B. Frank (1991) The 'epiligament' of the rabbit medial collateral ligament: a quantitative morphological study. Connect Tissue Res 27: 33-50.

Close, J.R. (1956) Some applications of the functional anatomy of the ankle joint. J Bone Joint Surg Am 38:761-781.

Cooper, D., F. Farr (1978) Ankle rehabilitation using the ankle disk. Physician Sports Med, pp. 6.

De Avila, G.A., B.L. O’Connor, D.M. Visco, T.D. Sisk (1989) The mechanoreceptor innervation of the human fibular collateral ligament. J Anat 162: 1-7.

Del Valle, M.E., S.F. Harwin, A. Maestro, A. Murcia, J.A. Vega (1998) Immunohistochemical analysis of mechanoreceptors in the human posterior cruciate ligament: a demonstration of its proprioceptive role and clinical relevance. J Arthroplasty 13: 916922.

Denti, M., Monteleone M., Berardi A., Panni A.S. (1994) Anterior cruciate ligament mechanoreceptors: histologic studies on lesions and reconstruction. Clin Orthop Relat Res, pp. 29-32.

Frank, C.B. (2004) Ligament structure, physiology and function. J Musculoskelet Neuronal Interact 4: 199-201.

-Freeman, M.A., M.R. Dean, I.W. Hanham (1965) The etiology and prevention of functional instability of the foot. J Bone Joint Surg Br 47: 678-685.

Freeman, M.A., B. Wyke (1967a) The innervation of the ankle joint: an anatomical and histological study in the cat. Acta Anat (Basel) 68: 321-333.

Freeman, M.A., B. Wyke (1967b) The innervation of the knee joint: an anatomical and histological study in the cat. J Anat 101: 505532 .
Glencross, D., E. Thornton (1981) Position sense following joint injury. J Sports Med Phys Fitness 21: 23-27.

Gómez-Barrena, E., E. Martinez-Moreno, R. Ballesteros Masso, D. Martinez Perez, L. Munuera Martinez (1999) Gold chloride technique to study articular innervation: a protocol validated through computer-assisted colorimetry. Histol Histopathol 14: 69-79.

Hagert, E. (2008) Wrist ligaments - innervation patterns and ligamento-muscular reflexes; thesis for doctoral degree (PhD), Karolinska Institutet, Stockholm.

Hagert, E. (2010) Proprioception of the wrist joint: a review of current concepts and possible implications on the rehabilitation of the wrist. J Hand Ther 23: 2-16.

Hagert, E., S. Forsgren, B.O. Ljung (2005) Differences in the presence of mechanoreceptors and nerve structures between wrist ligaments may imply differential roles in wrist stabilization. J Orthop Res 23: 757-763.

-Hagert, E., M. Garcia-Elias, S. Forsgren, B.O. Ljung (2007) Immunohistochemical analysis of wrist ligament innervation in relation to their structural composition. J Hand Surg Am 32: 30-36.

Hagert, E., J. Lee, A.L. Ladd (2012) Innervation patterns of thumb trapeziometacarpal joint ligaments. J Hand Surg Am 37: 706-714 e701.

Hagert, E., B.O. Ljung, S. Forsgren (2004) General innervation pattern and sensory corpuscles in the scapholunate interosseous ligament. Cells Tissues Organs 177: 47-54.

Hertel, J. (2002) Functional anatomy, pathomechanics, and pathophysiology of lateral ankle instability. J Athl Train 37: 364-375.

Ivie, T.J., R.C. Bray, P.T. Salo (2002) Denervation impairs healing of the rabbit medial collateral ligament. J Orthop Res 20: 990-995.

Johansson, H., P. Sjölander, P. Sojka (1991) A sensory role for the cruciate ligaments. Clin Orthop Relat Res, pp. 161-178.

Karlsson, J., G.O. Andreasson (1992) The effect of external ankle support in chronic lateral ankle joint instability: an electromyographic study. Am J Sports Med 20: 257-261.

Kennedy, J.C., I.J. Alexander, K.C. Hayes (1982) Nerve supply of the human knee and its functional importance. Am J Sports Med 10: 329-335.

Koch, B., G. Kurriger, R.A. Brand (1995) Characterisation of the neurosensory elements of the feline cranial cruciate ligament. J Anat 187: 353-359.

Konradsen, L., J.B. Ravn (1991) Prolonged peroneal reaction time in ankle instability. Int J Sports Med 12: 290-292.

Krauspe, R., M. Schmidt, H.G. Schaible (1992) Sensory innervation of the anterior cruciate ligament: an electrophysiological study of the response properties of single identified mechanoreceptors in the cat. J Bone Joint Surg Am 74: 390-397.
Kwan, M.M., J.C. Close, A.K. Wong, S.R. Lord (2011) Falls incidence, risk factors, and consequences in Chinese older people: a systematic review. J Am Geriatr Soc 59: 536-543.

Lee, J., A. Ladd, E. Hagert (2012) Immunofluorescent triple-staining technique to identify sensory nerve endings in human thumb ligaments. Cells Tissues Organs 195: 456-464,

Lin, Y.T., R.A. Berger, E.J. Berger, K. Tomita, J.Y. Jew, C. Yang, K.N. An (2006) Nerve endings of the wrist joint: a preliminary report of the dorsal radiocarpal ligament. J Orthop Res 24: $1225-1230$

Michelson, J.D., C. Hutchins (1995) Mechanoreceptors in human ankle ligaments. J Bone Joint Surg Br 77: 219-224.

Moraes, M.R., M.L. Cavalcante, J.A. Leite, F.V. Ferreira, A.J. Castro, M.G. Santana (2008) Histomorphometric evaluation of mechanoreceptors and free nerve endings in human lateral ankle ligaments. Foot Ankle Int 29: 87-90.

Morisawa, Y. (1998) Morphological study of mechanoreceptors on the coracoacromial ligament. J Orthop Sci 3: 102-110.

Munn, J., S.J. Sullivan, A.G. Schneiders (2010) Evidence of sensorimotor deficits in functional ankle instability: a systematic review with meta-analysis. J Sci Med Sport 13: 2-12.

Newton, R.A. (1982) Joint receptor contributions to reflexive and kinesthetic responses. Phys Ther 62: 22-29.

Pankovich, A.M., M.S. Shivaram (1979) Anatomical basis of variability in injuries of the medial malleolus and the deltoid ligament. 1. Anatomical studies. Acta Orthop Scand 50: 217-223.

Peter, R.E., R.M. Harrington, M.B. Henley, A.F. Tencer (1994) Biomechanical effects of internal fixation of the distal tibiofibular syndesmotic joint: comparison of two fixation techniques. J Orthop Trauma 8: 215-219.

Rammelt, S., H. Zwipp, R. Grass (2008) Injuries to the distal tibiofibular syndesmosis: an evidence-based approach to acute and chronic lesions. Foot Ankle Clin 13: 611-633, vii-viii.

Reimann, R., F. Anderhuber (1980) Kompensationsbewegungen der Fibula, die durch die Keilform der Trochlea tali erzwungen werden. Acta Anat (Basel) 108: 60-67.

Rein, S., T. Fabian, H. Zwipp, M. Mittag-Bonsch, S. Weindel (2010) Influence of age, body mass index, and leg dominance on functional ankle stability. Foot Ankle Int 31: 423-432.

Richie, D.H. Jr. (2001) Functional instability of the ankle and the role of neuromuscular control: a comprehensive review. J Foot Ankle Surg 40: 240-251.

Runge, M. (2002) Diagnostik des Sturzrisikos bei älter werdenden Menschen. Ther Umsch 59: 351-358.

Schmidt, H.M. (1978) Gestalt und Befestigung der Bandsysteme im Sinus und Canalis tarsi des Menschen. Acta Anat (Basel) 102: 184194. 
-Schultz, R.A., D.C. Miller, C.S. Kerr, L. Micheli (1984) Mechanoreceptors in human cruciate ligaments: a histological study. J Bone Joint Surg Am 66: 1072-1076.

-Schutte, M.J., E.J. Dabezies, M.L. Zimny, L.T. Happel (1987) Neural anatomy of the human anterior cruciate ligament. J Bone Joint Surg Am 69: 243-247.

-Solomonow, M. (2006) Sensory-motor control of ligaments and associated neuromuscular disorders. J Electromyogr Kinesiol 16: 549 567.

Soule, J.D. (1962) Direct staining of reticular fibers with gold chloride. Stain Technol 37: 31-34.
Spalazzi, J.P., J. Gallina, S.D. Fung-Kee-Fung, E.E. Konofagou, H.H. Lu (2006) Elastographic imaging of strain distribution in the anterior cruciate ligament and at the ligament-bone insertions. J Orthop Res 24: 2001-2010.

Stagni, R., A. Leardini, J.J. O’Connor, S. Giannini (2003) Role of passive structures in the mobility and stability of the human subtalar joint: a literature review. Foot Ankle Int 24: 402-409.

Takebayashi, T., T. Yamashita, Y. Minaki, S. Ishii (1997) Mechanosensitive afferent units in the lateral ligament of the ankle. J Bone Joint Surg Br 79: 490-493.

Teramoto, A., H. Kura, E. Uchiyama, D. Suzuki, T. Yamashita (2008) Three-dimensional analysis of ankle instability after tibiofibular syndesmosis injuries: a biomechanical experimental study. Am J Sports Med 36: 348352.
Tomita, K., E.J. Berger, R.A. Berger, J. Kraisarin, K.N. An (2007) Distribution of nerve endings in the human dorsal radiocarpal ligament. J Hand Surg Am 32: 466-473.

Tropp, H., P. Odenrick (1988) Postural control in single-limb stance. J Orthop Res 6: 833-839.

Ulfhake, B., E. Bergman, B.T. Fundin (2002) Impairment of peripheral sensory innervation in senescence. Auton Neurosci 96: 43-49.

Zwipp, H. (1986) Die anterolaterale Rotationsinstabilität des oberen Sprunggelenkes. Hefte Unfallkd 177: 1-179.

Zwipp, H. (1994): Chirurgie des Fusses. New York, Springer. 